\title{
A new method of vascular point detection using Artificial Neural Network
}

\author{
S.Kader, A.M Aibinu, M.J.E Salami \\ Mechatronics Engineering Department \\ International Islamic University \\ Kuala Lumpur Malaysia
}

\begin{abstract}
Vascular intersection is an important feature in retina fundus image (RFI). It can be used to monitor the progress of diabetes hence accurately determining vascular point is of utmost important. In this work a new method of vascular point detection using artificial neural network model has been proposed. The method uses a $5 \times 5$ window in order to detect the combination of bifurcation and crossover points in a retina fundus image. Simulated images have been used to train the artificial neural network and on convergence the network is used to test (RFI) from DRIVE database. Performance analysis of the system shows that ANN based technique achieves 100\% accuracy on simulated images and minimum of $92 \%$ accuracy on RFI obtained from DRIVE database.
\end{abstract}

Keywords-Diabetic Retinopathy,Retina,Vascular points, Artificial Neural Network

\section{INTRODUCTION}

The retina is made up of thin layers of blood vessels and located at the back of the eye. Eye needs to receive constant blood supply so that eye can function properly as well as to prevent from loss of vision. Consequently, blood supplies oxygen and nutrient in order to maintain healthy eye. In general overview, there are two types of retinal vessels in the retina namely retinal arteries and retinal veins. The retinal arteries carry all the blood supply needed to the nerve cells whereas the retinal veins carry the blood away from the nerve cells. The artery blood vessels and vein blood vessels are separated by retina capillary wall which act as a barrier in the retina. Thus, the abnormal retinal vessel will lead to eye diseases such as branch retinal vein occlusion, central retinal vein occlusion and neovascularization.

The artery and vein crossover, make the blood pressure increase in the retinal artery and compress the retinal vein at the vein artery cross over points and sub consequently thickened artery will interrupt the blood flow as well as increase the pressure inside the retinal vein. The swollen veins will cause blockage in the retina and lead to branch retinal vein occlusion or abnormal vein artery crossover points [1]. Thus blood vessels are also one of the important features used for diagnosing diabetic retinopathy in retina fundus images.

The information about the changes in the blood vessels appearance in the retina is used in [2] to diagnose certain eye related diseases such as diabetic retinopathy, hypertension and arteriosclerosis. Consequently, the automated detection of retinal blood vessels in fundus image will assist the ophthalmologist to examine for any abnormalities or changes occur in the blood vessels.

Therefore, the importance of retinal vasculature in the retinal identification for the biometric application is highlighted in [3]. The stem of retinal vascular is unique for each individual as well as cannot be replaced or changed with artificial retinal vascular stem. Likewise, proposed in [4], illustrated image matching technique and the usage of bifurcation and crossover point as feature vectors used to match with the corresponding image. The concept of retinal image matching was applied in the biometric security application.

\section{LITERATURE REVIEW}

There are numerous techniques used for blood vessel segmentation in retinal images. They were reported in [5].The vascular network is in the form of retinal vessel tree structure. The vascular network or retinal blood vessels associated with its features such as bifurcation and crossover. The bifurcation points are defined as a point in the blood vessels. They are divided into smaller blood vessels whereas the crossover points are defined as a point where the two blood vessels are cross each other.

Therefore there are various techniques used to detect bifurcation and crossover points in the vascular network. For example, [6] introduced modified cross number point method to detect bifurcation and vein - artery cross over points in fundus images. There are three different techniques involved in this work such as digital image processing, fuzzy logic and artificial neural network. Thus, the modified cross number obtained from the digital image processing technique and neural network technique to detect the bifurcation and cross points. The simple cross number $(\mathrm{SCN})$ method used a $3 \times 3$ window with 8 neighbouring pixels whereas in modified cross number $(\mathrm{MCN})$ method used a $5 \times 5$ window with 16 neighbouring pixels. Result showed that artificial neural network technique superior to simple cross point (SCN) method and modified cross point (MCN) method. The false detection rate was found in the modified cross number (MCN) method.

Some improvement has been done for efficient detection of bifurcation and crossover points in [7].Thus in this work, the 
bifurcation points were sampled so that the over counting pixels were resolved.

Another method used in [8] is combined cross number (CCN) method where the simple cross number (SCN) method and modified cross number (MCN) method applied in this work. This new hybrid approach is the improvement from the previous work. The combined cross number $(\mathrm{CCN})$ also used $5 \times 5$ window but there are some changes in terms of window's arrangement. The proposed method reduced the false detection rate compared to the previous work.

Furthermore, digital signal and image processing techniques has been proposed [1] for vein -artery crossover points detection. This work needs some improvement to avoid misclassification on the normal cross over points and abnormal cross over point in fundus image.

Besides that, there are four different types of filters used in [9] to detect bifurcation and crossover points by extracting the blood vessels features. This proposed method was not an automated process which does not involved with supervised learning technique.

On the other hand, the supervised learning technique is used in [10] to detect bifurcation and crossover points in fundus images. The extracted image were used for edge extraction by using Genetic Algorithm, and the edge images were used in the skeleton process to eliminate all isolated points as well as the edge images were fused with skeleton images in the tracking algorithm in the last stage.

Other than that, the registration retina image approach using Bayesian Hough Transform for the detection of bifurcation points in the vessels were investigated in [11]. The Hough Transform technique is used to match pathology images with the reference images. The major drawback of this proposed method is, time taken for registering the retinal image has increased.

Moreover, the local thresholding entropy method for efficient blood vessels detection has been proposed in [2]. The method detection used in this work tends to misclassify other lesions as blood vessels.

Two different approaches were involved in the analysis of feature points in [12]. The first approach is the detection of feature points whereas the second approach is the classification of feature points. The improvement that has been done in this work compared with the existing techniques where the segmentation step was implemented before the preprocessing step. Hence, any connectivity loss or any misclassification of feature points that usually occurred in the pre-processing step has been resolved. Some points such as acute crossover points were omitted in the classification step in this work due to the number of vessels associated with the points are used instead of used vessels features.

The detection of junction candidate points and detection of location refinement and junction branch has been proposed in [13]. Even though the proposed method is able to detect very tiny branches in the retina image but it still need some improvement in the intensity contrast between foreground and background image.

In addition, the multimodal registration retinal image technique based on self-organizing map was introduced in [14]. The self-organizing map (SOM) theory was used for automated detection of bifurcation points between the reference image and desired image. Result showed high accuracy is obtained in the proposed method.

As a result various techniques had been reviewed as above for the detection of bifurcation and cross over points in fundus image. However, many of the researches have attempted to use image processing techniques and very few of them used artificial intelligent approach in their work for the detection of bifurcation and crossover points in fundus image.

\section{METHODOLOGY}

In this section, a new method of $\mathrm{BP}$ and $\mathrm{CP}$ detection using ANN based MCN and CCN is proposed. A 25-10-1 (i.e. 25 input nodes, 10 nodes in the hidden layer and 1 node in the output layer) ANN has been used for the detection of ANN. Samples of BP and CP points were used to train the neural network using back propagation algorithm explained in (1) to (11).Fig. 1 shows the flowchart of $\mathrm{CCN}$ technique embedded with ANN.

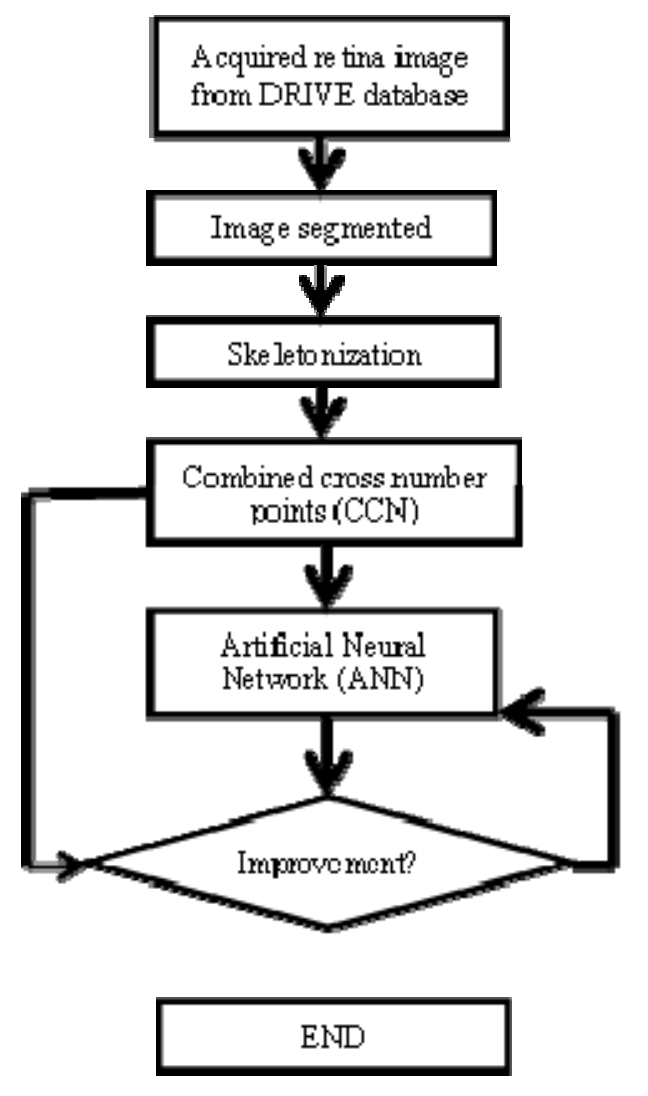

Figure 1. The Flowchart of Improvement in CCN Technique 


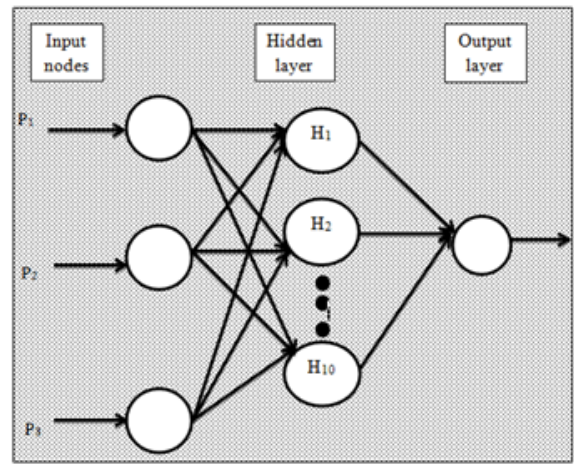

Figure 2. ANN based parametric model diagram showing synaptic weight connection

The error signal at the actual output $e(n)$ is given as

$$
e(n)=T(n)-y(n)
$$

where $T(n)$ is defined as a desired output and $y(n)$ is defined as an actual output.

The minimized squared error is given as

$$
\xi=\frac{1}{N} \sum_{n=1}^{N} e(n)^{2}
$$

where $\mathrm{N}$ is the set of training examples, $\xi$ is the error gradient in the output layer. The updating weight at the output layer is given as

$$
w(n+1)=w(n)+\Delta w(n)
$$

Thus the weight correction is defined as

$$
\Delta w(n)=-\mu \nabla_{w} \xi=-\mu \frac{\partial \xi}{\partial w_{j n}}
$$

where $\mu$ is denoted as learning rate, $\nabla_{w} \xi$ is defined as gradient descent objective function, and is given as

$$
\frac{\partial \xi}{\partial w_{j n}}=\frac{\partial \xi}{\partial y} \frac{\partial y}{\partial u} \frac{\partial u}{\partial w_{j n}}
$$

Let

$$
\begin{aligned}
& \partial(n)=-\frac{\partial \xi}{\partial y} \\
& y(n)=\digamma(u(n))
\end{aligned}
$$

$$
u(n)=\sum_{j} X_{j n} w_{j n}
$$

Where $\mathrm{X}_{\mathrm{jn}}$ is denoted as a total input value of neuron $j n$

$$
\frac{\partial u}{\partial w_{j n}}=X_{j n}
$$

Therefore (3.5) becomes

$$
\frac{\partial \xi}{\partial w_{j n}}=\nabla{ }_{w} \xi=-X_{j n} \digamma^{\prime}(u(n)) \partial(n)
$$

Thus the output layer for update weight is given as

$$
\begin{aligned}
w(n+1) & =w(n)+\mu X \underset{j n}{ } \digamma^{\prime}(u(n)) \partial(n) \\
& =w(n)+\Delta w(n)
\end{aligned}
$$

Once the ANN converges during the training, the weights and the adaptive coefficients of the ANN are extracted using the method proposed in [15].The newly proposed method involve the use of parametric modelling approach where the weights and the adaptive coefficients of the ANN are used in the computation of the parametric coefficients $a_{k}$ given in

$$
\mathrm{y}(\mathrm{n})=-\mathrm{g}_{\mathrm{k}} \mathrm{g}{ }_{1} \mathrm{a}_{\mathrm{n}} F_{\mathrm{k}}+\mathrm{b}_{0} \mathrm{x}(\mathrm{n})
$$

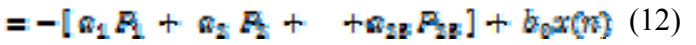

The computation and the derivation of $a_{k}$ have been discussed extensively in [16]. This involves computing the output of the ANN shown in Figure 3.5. The output is expressed as

$$
y(n)=\alpha F\left(\sum_{[=1}^{M} w_{\left[1 \theta_{2}+\bar{B}_{02}\right.}\right)
$$

where $M$ is the number of neurons in the hidden layer, $w_{11}$ is the weight connecting node 1 in the hidden layer to output layer, $h_{01}$ is the bias term of output neuron, $w_{1}$ is the first output of the hidden node 1 and $\alpha$ is the adaptive coefficient of the linear output activation function. The hidden node output $w_{1}$ can be expressed as

$$
y(n)=\beta_{1} F\left(\sum_{n=1}^{D} v_{k i \theta_{1}} y(n-k)+\beta_{0 i}\right)
$$

where $v_{k l}$ is the weight connecting input node $\mathrm{k}$ to hidden node $1, g_{01}$ is the bias of the hidden node 1 and $\beta_{1}$ is the adaptive coefficient of hidden node linear activation function. Substituting (13) into (14) gives (15) and the output coefficients can be computed using (16). 


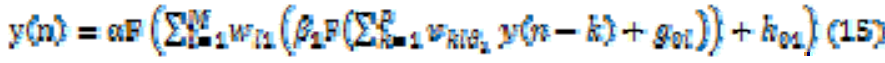

$$
\begin{aligned}
& a_{i=}=\sum_{n=1}^{M} w_{i 1} v_{k i} \beta_{1}
\end{aligned}
$$

Thus a new parametric method of computing the coefficients of the model required to determine $\mathrm{BP}$ and $\mathrm{CP}$ has been proposed in this work.

\section{RESULTS AND PERFORMANCE ANALYSIS}

In the detection of BP and CP termed vascular point (VP), as required for $\mathrm{ANN}$ technique, 54 sample images of BP, 54 sample images and 50 samples of non-vascular points were simulated using digital image processing technique, sample of which are as shown in Fig. 3 These were used to train neural network hence the ANN based VP detection consist of 150 pairs of input and output data.

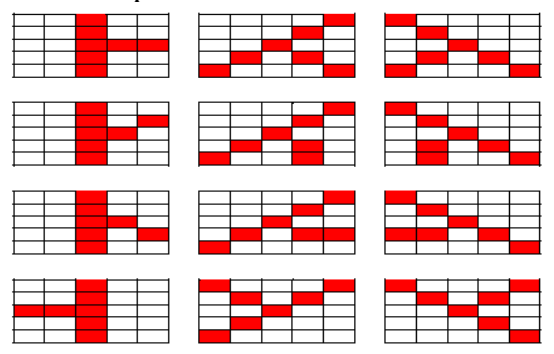

a.Simulated BP points

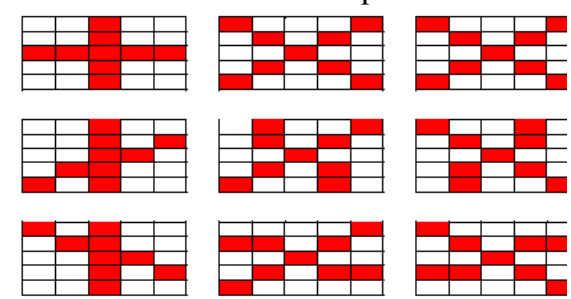

b. simulated CP points

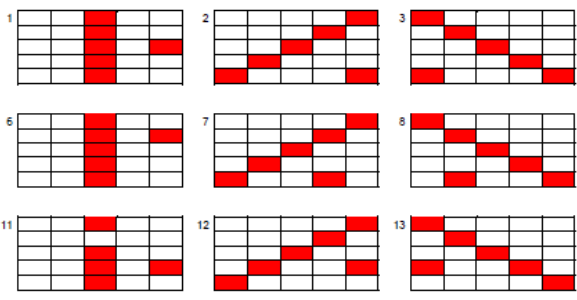

c. Samples on non-CP/BP (VP) points.

Figure 3. (a) Samples of simulated BP points. (b) Samples of simulated CP points. (c) Samples of simulated non-BP/CP (VP) points.

Various ANN configurations were tested in this work and it was observed that 25 inputs, two layer ANN with 10 neurons at the hidden layer and one neuron at the output layer is appropriate for this work. The pair of simulated data was used as training input for the neural network and the detail analysis have been previously discussed in the methodology section. Mean square error (MSE) for training data is as shown in Fig. 4.It can be observed that the training was perfectly done as the value of MSE obtained ranges from $1.533 \times 10^{-9}$ to $1.473 \times 10^{-7}$.

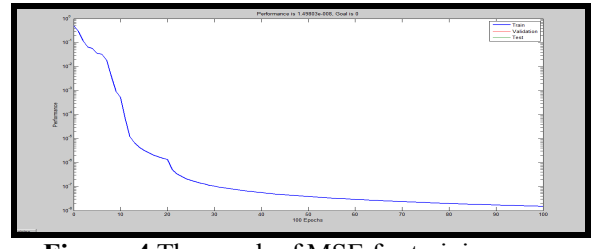

Figure 4.The graph of MSE for training error

Upon convergence and completion of the training, testing of the system was conducted. For each test case in Table 1, the performances were obtained over an average of 3-10 runs so as to check for precision and variation. It was however observed that similar results were obtained in each of the runs hence having a variance of 0 .

Table 1

The performance for each test case

\begin{tabular}{|l|l|l|l|l|l|l|l|}
\hline Method & P & TP & FP & FN & PR & FPR & TPR \\
\hline Run-1 & 150 & 150 & 0 & 0 & 1.00 & 0.00 & 1.00 \\
\hline Run-11 & 150 & 150 & 0 & 0 & 1.00 & 0.00 & 1.00 \\
\hline Run-21 & 150 & 150 & 0 & 0 & 1.00 & 0.00 & 1.00 \\
\hline Run-40 & 150 & 150 & 0 & 0 & 1.00 & 0.00 & 1.00 \\
\hline
\end{tabular}

Table 2

Detection results and comparison between other methods and ANN technique

\begin{tabular}{|l|l|l|l|}
\hline Method & $\begin{array}{l}\text { Image 6 } \\
(\text { sample 1) }\end{array}$ & $\begin{array}{l}\text { Image } \\
16(\text { sample 2) }\end{array}$ & $\begin{array}{l}\text { Image } \\
18(\text { sample 3) }\end{array}$ \\
\hline SCN 3X3 & 64 & 113 & 53 \\
\hline CCN 5x5 & 61 & 96 & 45 \\
\hline MCN 5x5 & 64 & 106 & 46 \\
\hline ANN & 62 & 99 & 49 \\
\hline
\end{tabular}

In conclusion, ANN technique record $100 \%$ accuracy, precision and TPR over training data set in this work. The trained ANN system was now used to detect VP in RFI using Drive database. Table 2 shows parts of the results obtained when the proposed algorithm was tested on RFI in Drive database obtained from [16]. Three images were selected at random for reporting in this section, however the results obtained for 15 images in DRIVE database. For the selected image, the ground truth of Image 6 shows that there are 60 vascular points (VP) in the image. These VP are the positive points to be detected by the algorithm while undetected VP are termed the false negative (FN). Detail analysis of the image as shown in Fig. 5 and Table 3.

Table 3

Vascular point Detection for Image 6

\begin{tabular}{|l|l|l|l|l|l|l|l|l|}
\hline Method & $\begin{array}{l}\text { Image } \\
6\end{array}$ & P & TP & FP & FN & PR & FPR & TPR \\
\hline $\begin{array}{l}\text { SCN } \\
\text { 3X3 }\end{array}$ & 64 & 60 & 60 & 4 & 2 & 0.9375 & 0.004 & 1.00 \\
\hline $\begin{array}{l}\text { CCN } \\
\mathbf{5 x 5}\end{array}$ & 61 & 60 & 59 & 2 & 0 & 0.9672 & 0.002 & 0.983 \\
\hline $\begin{array}{l}\text { MCN } \\
\mathbf{5 x 5}\end{array}$ & 64 & 60 & 60 & 4 & 0 & 0.9375 & 0.004 & 1.00 \\
\hline ANN & 62 & 60 & 59 & 2 & 0 & 0.9672 & 0.002 & 0.9833 \\
\hline
\end{tabular}


In the detection of vascular point as shown in Table 2, the precision of the newly introduced ANN method is comparative with the existing technique. The SCN shows the least results while the ANN and the CCN method shows better performance as compared with the other techniques. This shows that precision obtained in this work is comparable with those reported in the literature [6-9].Fig. shows the results obtained for each of the methods evaluated in this work. As observed from Table 3, the FP for SCN method is the highest while ANN and CNN recorded the lowest FP. Similarly, SCN and MCN accurately detect all the positive points in the image.The case of undetected point was analyzed at it was observed that the pattern for that point was not in the training database images, hence inclusion of it for subsequent training images.

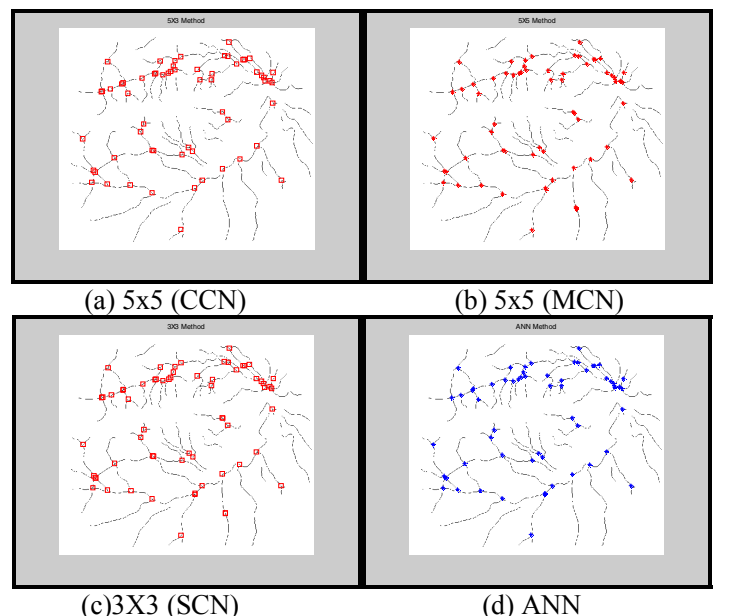

Figure.5. Results obtained for Image 6 in Drive Database using (a) $3 \times 3$ (SCN) method (b) $5 \times 5(\mathrm{CCN})(\mathrm{c}) 5 \times 5(\mathrm{MCN})$ and (d) ANN

Similar to what was obtained in the first test image, results obtained using sample image 16, using SCN method gives 113 vascular points while using CCN method 96 vascular points were detected. In addition, MCN and ANN 106 and 99 were detected respectively. Detail analysis of the image shows that 15 false positive were detected using SCN method, followed by MCN, 9 false positive were detected, while $\mathrm{CCN}$ method, 8 false positive were detected and ANN technique, only 1 false positive was detected. The PR, FPR and TPR were calculated for each of the methodology and it was observed that ANN gives the best PR TPR and lowest FPR. This information are contained in Table 4 and Fig. 6. shows the detailed analysis showing false positive detection in each of the methodology.

\section{Table 4}

Vascular point Detection for Image 16

\begin{tabular}{|l|l|l|l|l|l|l|l|l|}
\hline Method & $\begin{array}{l}\text { Image } \\
16\end{array}$ & P & TP & FP & FN & PR & FPR & TPR \\
\hline $\begin{array}{l}\text { SCN } \\
\mathbf{3 X 3}\end{array}$ & 113 & 98 & 98 & 15 & 0 & 0.8673 & 0.015 & 1.00 \\
\hline $\begin{array}{l}\text { CCN } \\
\mathbf{5 x 5}\end{array}$ & 96 & 98 & 88 & 8 & 0 & 0.9167 & 0.008 & 1.00 \\
\hline $\begin{array}{l}\text { MCN } \\
\mathbf{5 x 5}\end{array}$ & 106 & 98 & 98 & 8 & 0 & 0.9245 & 0.008 & 1.00 \\
\hline $\mathbf{A N N}$ & 99 & 98 & 98 & 1 & 1 & 0.9899 & 0.001 & 1.00 \\
\hline
\end{tabular}

Similarly, the detection of vascular point for image 16 is shown in Table 4. The TPR obtained for all the methods are consistently high and it is worth noting that the FN obtained for ANN method is consistently low when compared to other techniques. Hence, ANN method can be used for further detection of VP in RFI.
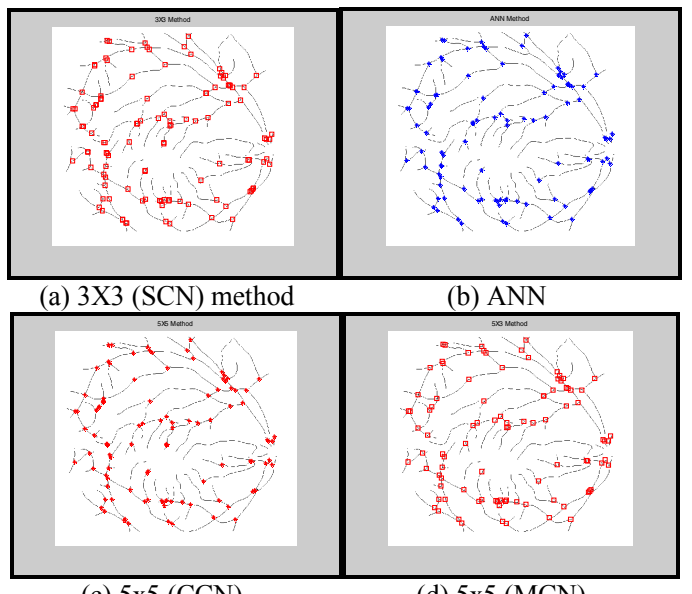

(c) $5 \times 5(\mathrm{CCN})$

(d) $5 \times 5(\mathrm{MCN})$

Figure 6. Results obtained for Image 16 in Drive Database using (a) $3 \times 3$ (SCN) method (b) ANN (c) $5 \times 5(\mathrm{CCN})$ and (d) $5 \times 5(\mathrm{MCN})$

Lastly, in column 3 of Table 2, using sample image 18 for discussion, SCN was able to detect 53 VP while using CCN method 45 points were detected. Similarly for MCN and ANN 46 and 49 were detected respectively. Detail analysis of the image as shown in Table 5 and Fig. 7 shows that 9 false positive were detected using $\mathrm{SCN}$, whereas 2 false positive were detected in ANN, followed by MCN, 3 false positive were detected and lastly, 1 false positive was detected in CCN.
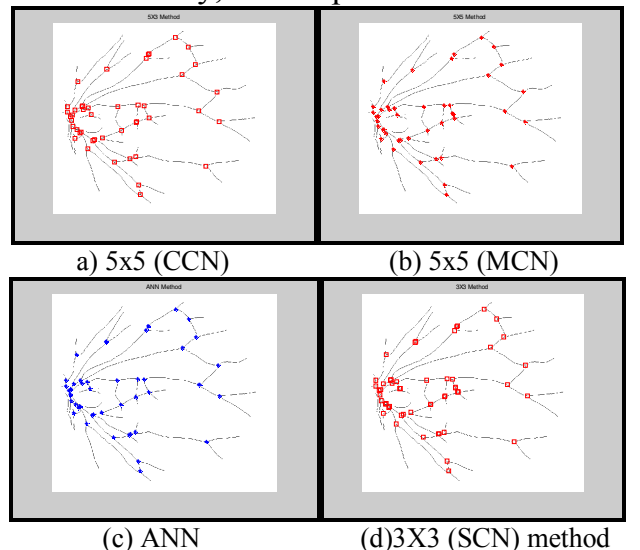

Figure 7. Results obtained for Image 18 in Drive Database using (a) $5 \times 5$ $(\mathrm{CCN})(\mathrm{b}) 5 \times 5(\mathrm{MCN})(\mathrm{c}) \mathrm{ANN}$ and (d) $3 \mathrm{X} 3(\mathrm{SCN})$ method

Table 5

Vascular point Detection for Image 18

\begin{tabular}{|l|l|l|l|l|l|l|l|l|}
\hline Method & $\begin{array}{l}\text { Image } \\
\mathbf{1 8}\end{array}$ & $\mathbf{P}$ & $\mathbf{T P}$ & $\mathbf{F P}$ & $\mathbf{F N}$ & $\mathbf{P R}$ & FPR & TPR \\
\hline $\begin{array}{l}\text { SCN } \\
\mathbf{3 X 3}\end{array}$ & 53 & 44 & 44 & 9 & 0 & 0.8673 & 0.0000 & 1.00 \\
\hline $\begin{array}{l}\mathbf{C C N} \\
\mathbf{5 x 5}\end{array}$ & 45 & 44 & 44 & 1 & 0 & 0.9167 & 0.0001 & 1.00 \\
\hline $\begin{array}{l}\mathbf{M C N} \\
\mathbf{5 x 5}\end{array}$ & 46 & 44 & 44 & 3 & 2 & 0.9245 & 0.0002 & 1.00 \\
\hline $\mathbf{A N N}$ & 45 & 44 & 44 & 1 & 1 & 0.9899 & 0.0001 & 1.00 \\
\hline
\end{tabular}


Among the reported techniques for detecting VP tested in this work, it was observed that the use of the proposed ANN technique took the longest time for completion (TOC). Typical obtained in detecting VP is as shown in Table 6

Table 6

Time taken for completion

\begin{tabular}{|l|l|}
\hline Method & $\begin{array}{l}\text { TOC } \\
\text { (seconds) }\end{array}$ \\
\hline SCN 3X3 & 9.8143 \\
\hline CCN 5x5 & 9.4413 \\
\hline MCN 5x5 & 9.5967 \\
\hline ANN & 47.8506 \\
\hline
\end{tabular}

The reason for the long time for completion of ANN method is that, the reported TOC include training and testing time of the algorithm. Without considering the ANN training time, the TOC reported is just 17.4393 seconds.

\section{CONCLUSION}

In this work, a new method of detecting VP has been proposed and evaluated in this work. The VP detection achieved 100\% accuracy over training data recorded and minimum accuracy of $96.23 \%$ as well as maximum of $100 \%$ using Drive database system. It has recorded significant performance with other technique; hence it can use for monitoring and diagnosis of diabetes.

\section{REFERENCES}

[1] Aibinu.A.M, Iqbal.M.I, Shafie.A.A, Salami.M.J.E\& Nilsson.M, Vascular intersection detection in retina fundus images using a new hybrid approach. Journal on Computers in Biology and Medicine, 40(1) (2010) 81-89

[2] T. Chanwimaluang, and G. Fan, "An Efficient Blood Vessel Detection Algorithm for Retinal Images Using Entropy Thresholding", Proceedings of the 2003 International Symposium on Circuits and Systems, Bangkok, Thailand, vol.5, pp.V-21-V-24, May2003.

[3] C. Köse, C. Ikibas, A personal identification system using retinal vasculature in retinal fundus images, Expert Systems with Applications 38 (2011) 13670-13681.

[4] A.Bhuiyan,Lamoureux.EB.Nath,K.Ramamohanara, T.Y.Wong, Retinal Image Matching Using Hierarchical Vascular Features, Hindawi Publishing Corporation Computational Intelligence and Neuroscience (2011)

[5] M.M. Fraz, et al., Blood vessel segmentation methodologies in retinal images - A survey, Comput. MethodsProgramsBiomed.(2012), http://dx.doi.org/10.1016/j.cmpb.2012.03.009, in press.

[6] Iqbal.M.I,Aibinu.A.M,Tijani.I.B,Salami.M.J.E.(2008) Detection of Vascular Intersection in Retina Fundus Image Using Modified Cross Point Number and Neural Network Technique.Proceeding of the International
Conferences on Computer and Communication Engineering, pp.241-246.

[7] Turetkan, E.,Blum,C.,Gonzalez, G.,\& Fua,P. Reconstructing Geometrically Consistent Tree Structures from Noisy Images.

[8] A. M. Aibinu, M. I. Iqbal, M. Nilsson and M. J. E Salami,. "Automatic Diagnosis of Diabetic Retinopathy from Fundus Images Using Digital Signal and Image Processing Techniques", Proc. International Conference on Robotics, Vision, Information, and Signal Processing, Penang, Malaysia, pp. 510-515, Nov. 2007.

[9] V. Bevilacqua, S. Cambo, L. Cariello and G. Mastronardi "“A Combined Method to Detect Retinal Fundus Features", European Conference on Emergent Aspects in Clinical Data Analysis, Pisa, Italy,Sept.2005.

[10] V. Bevilacqua, S. Cambo, L. Cariello and G. Mastronardi “".(2007) Retinal fundus hybrid analysis based on soft computing algorithms. Communications to SIMAI Congress, 2.

[11] F. Zana, J.C. Klein, A multimodal registration algorithm of eye fundus images using vessels detection and Hough transform, IEEE Transactions on Medical Imaging 18 (1999) 419-428.

[12] Calvo,D., Ortega, M., Penedo, M. G.,\& Rouco, J.(2011). Automatic detection and characterisation of retinal vessel tree bifurcations and crossovers in eye fundus images. Computer methods and programs in bio medicine, 28-38.

[13] Su, R.,Sun,C.,\& Pham, T.D.(2012). Junction detection for linear structures based on Hessian, correlation and shape information. Pattern Recognition 45, 3695-3706

[14] Matsopoulos G. K., Asvestas P. A., Mouravliansky N.A.and Delibasis K. K.(2004,December). Multimodal Registration of Retinal Images Using Self Organizing Maps.IEEE Transactions On Medical Imaging, 23(12).

[15] Aibinu, A.M., et al., Artificial neural network based autoregressive modeling technique with application in voice activity detection. Eng. Appl. Artif. Intel. (2012), http://dx.doi.org/10.1016/j.engappai.2012.05.012, in press.

[16] The DRIVE database accessed http://www.isi.uu.nl/Research/Databases/DRIVE, 2012. 